\title{
Prognostic value of red blood cell distribution width for intestinal resection in inflammatory bowel disease
}

\author{
Jiaojie Xue ${ }^{1}$, Min Zhang ${ }^{1}$, Yitao Zhang ${ }^{1}$, Zhichong Chen ${ }^{1}$, Shiyao Cheng ${ }^{1}$, Mao Ouyang ${ }^{1}$, \\ Min $\mathrm{Zhi}^{1}$, and Weijie Zeng ${ }^{1}$ \\ ${ }^{1}$ Sun Yat-sen University Sixth Affiliated Hospital
}

September 1, 2021

\begin{abstract}
INTRODUCTION: Inflammatory bowel disease (IBD) is a non-specific chronic inflammatory disease. Previous studies confirmed that inflammation plays an important role and red blood cell distribution width (RDW) is related to the activity of IBD. OBJECTIVES: To identify the prognosis value of RDW in the intestinal resection of IBD. PATIENTS AND METHODS: A total of 366 patients were included in this retrospective analysis, diagnosed with IBD between March 2012 and November 2014 in the Sixth Affiliated Hospital of Sun Yat-Sen University. All participants were followed up at least 5years. Patients' demographic characteristics and laboratory data were obtained from medical record within $24 \mathrm{~h}$ at first hospitalization. We compared the clinical features in the intestinal resection and non-intestinal resection groups. Kaplan-Meier estimates of the probability of intestinal resection post-admission time were computed, and compared with log-rank test according to stratification of RDW value. RESULTS: There were 93 patients in the intestinal resection group and 273 in the non- intestinal resection group. Significant higher RDW values were detected in the intestinal resection group $(\mathrm{P}<0.001)$. RDW was obviously related to indicators such as C-reactive protein $(\mathrm{p}=0.008)$. Univariate Cox regression analysis demonstrated that IBD type (Crohn's disease or ulcerative colitis) $(\mathrm{P}<0.001)$, RDW $(\mathrm{P}<0.001)$, C-reactive protein $(\mathrm{P}<0.001)$ and others were associated with intestinal resection probability. But only RDW [OR: 1.088 (95\% CI 1.009-1.173), $\mathrm{P}=0.03$ ] and IBD type $(\mathrm{P}=0.001)$ were independent predictors. CONCLUSIONS: RDW and IBD type were independent predictors of intestinal resection in IBD. KEY WORDS: inflammatory bowel disease, intestinal resection, red blood cell distribution width
\end{abstract}

Prognostic value of red blood cell distribution width for intestinal resection in inflammatory bowel disease

\section{ABSTRACT}

INTRODUCTION: Inflammatory bowel disease (IBD) is a non-specific chronic inflammatory disease. Previous studies confirmed that inflammation plays an important role and red blood cell distribution width $(\mathrm{RDW})$ is related to the activity of IBD.

OBJECTIVES: To identify the prognosis value of RDW in the intestinal resection of IBD.

PATIENTS AND METHODS: A total of 366 patients were included in this retrospective analysis, diagnosed with IBD between March 2012 and November 2014 in the Sixth Affiliated Hospital of Sun Yat-Sen University. All participants were followed up at least 5years. Patients' demographic characteristics and laboratory data were obtained from medical record within $24 \mathrm{~h}$ at first hospitalization. We compared the clinical features in the intestinal resection and non-intestinal resection groups. Kaplan-Meier estimates of the probability of intestinal resection post-admission time were computed, and compared with log-rank test according to stratification of RDW value.

RESULTS: There were 93 patients in the intestinal resection group and 273 in the non- intestinal resection group. Significant higher RDW values were detected in the intestinal resection group $(P<0.001)$. RDW 
was obviously related to indicators such as C-reactive protein $(\mathrm{p}=0.008)$. Univariate Cox regression analysis demonstrated that IBD type (Crohn's disease or ulcerative colitis) $(P<0.001)$, RDW $(P<0.001)$, C-reactive protein $(P<0.001)$ and others were associated with intestinal resection probability. But only RDW [OR: 1.088 (95\% CI 1.009-1.173), $\mathrm{P}=0.03]$ and IBD type $(\mathrm{P}=0.001)$ were independent predictors.

CONCLUSIONS: RDW and IBD type were independent predictors of intestinal resection in IBD.

KEY WORDS: inflammatory bowel disease, intestinal resection, red blood cell distribution width INTRODUCTION

Inflammatory bowel disease (IBD) is a chronic inflammatory disease, including Crohn's disease (CD) and ulcerative colitis (UC). The inflammatory markers are often elevated in IBD patients, such as C-reactive protein (CRP). However, there is significant heterogeneity in CRP response between CD and UC patients. CRP was significantly increased in CD patients, but only modest-to-absent increased in UC patients ${ }^{1,2}$. Therefore, CRP cannot assess the severity and activity of the disease well. The heterogeneity of inflammatory factors may partly explain different treatment strategies and outcomes.

So far, the treatment of IBD has been regarded as a challenge due to its extensive heterogeneity and extensive extraintestinal manifestations. It includes drug treatment and surgical treatment. The medications that induce remission include steroids and Tumor Necrosis Factor inhibitors ${ }^{3}$. The surgical treatment includes bowel resection, stricturoplasty or drainage of abscess. Approximately two-thirds of IBD patients will require surgical treatment during their lifetime ${ }^{3}$. The most common indications for intestinal resection in IBD patients are perforation, persistent obstruction, abscesses that cannot be drained percutaneously, and intractable bleeding ${ }^{3}$. Timely surgical treatment can improve the prognosis of patients who have failed conservative medications. Therefore, it is very important to determine which patients need surgical treatment. Various indicators have been used to assess the severity of IBD patients ${ }^{4}$. Unfortunately, there is no indicator that can accurately predict the prognosis of patients.

Red blood cell distribution width (RDW) is a simple method to measure the size distribution width of red blood cells. It is simple and easy to obtain. Some studies have shown that RDW is associated with disease activity in $\mathrm{IBD}^{5-8}$. In addition, studies have found that RDW can predict poor prognosis of colorectal cancer $^{9}$. However, the value of RDW in predicting the possibility of intestinal resection in IBD has not been studied. Therefore, we conducted a retrospective study to determine the prognostic value of RDW in IBD.

\section{PATIENTS AND METHODS}

\section{Study design and participants}

A total of 366 patients were diagnosed with IBD between October 2012 and November 2014 were selected for this study, and followed until May 2020. This study was approved by the institutional review board at the sixth affiliated Hospital of Sun Yat-Sen University. Written informed consent was waived by the Ethics Commission.

\section{Data collection}

Data including patient's demographic information, comorbidities, home medications, initial laboratory test, inpatient medications, treatments, and outcomes were collected from medical record. The results of the first blood laboratory test after admission was recorded. Follow-up data were collected by telephone interviews with patients or review of the patients' records. An automatic hematology analyzer was used to test routine blood indexes, and a Hitachi 7170-automatic biochemical analyzer was used to measure biochemical indicators.

\section{Definitions}

Disease activity was determined for patients with CD and UC with the Crohn's disease activity index and Mayo score, respectively. Patients with CD were divided into the following 3 groups according to the Crohn's disease activity index: (1) 150[?]mild activity $<220$; (2) 220[?] moderate activity $<450$; and severe 
activity[?]45010. The UC patients were divided into the following 3 groups according to the Mayo score: (1) $3[?]$ mild activity $<6$; (2) 6 [?]moderate activity $<10$, and 10[?] severe activity ${ }^{11,12}$.

\section{Statistical analysis}

Patients were divided into 3 groups, mild activity, moderate activity, and severe activity, according to the criteria mentioned above. The data were analyzed using the SPSS software, version 24.0 (SPSS Inc. Chicago, Illinois, United States). Continuous variables are presented as mean (SD) or medians (interquartile range $[\mathrm{IQR}]$ ), as appropriate. Categorical variables were summarized as numbers and percentage. Correlation between data sets was evaluated using Person correlation analysis. A receiver operating characteristic curve was generated to identify the optimal cutoff value of RDW for predicting intestinal resection. Time to event was defined as the time from diagnosis to the first time intestinal resection. Kaplan-Meier estimates were used to draw the cumulative incidence curves, compared by log-rank tests. Univariate Cox analyses were performed to identify predictors for the probability of intestinal resection. All variables with a $\mathrm{P}<0.05$ were then tested in a multivariate Cox analyses (forward LR procedure). A two-sided alpha of less than 0.05 was considered statistically significant.

\section{RESULTS}

\section{Patient characteristics}

A total of 366 patients with complete data were included in this study. The patient characteristics are reported in Table 1. The median (IQR) age of 366 patients with IBD was 30 (22-41) years, and $33.1 \%$ were female. These included $261 \mathrm{CD}$ patients and $105 \mathrm{UC}$ patients. $89 \mathrm{CD}$ patients (34.1\%) had received intestinal resection during follow-up, but only 4 UC patients $(3.8 \%)$ received intestinal resection therapy. Patients with CD are more likely to receive intestinal resection. The RDW was shown to be significantly higher in intestinal resection group as compared with non- intestinal resection group $(16.20 \%$ [3.24\%] vs. $14.76 \%$ [2.70\%], $\mathrm{p}<0.001$ ) (Table 1). Systolic blood pressure, platelet count, neutrophil ratio, lymphocyte ratio, C-reactive protein and high-sensitivity C-reactive protein differed significantly between the intestinal resection group and non- intestinal resection group $(\mathrm{p}<0.05)$. But, there were no significant differences in age, proportion of females, diastolic pressure, creatinine, uric acid, platelet distribution width and white blood cell count $(\mathrm{p}>0.05)$.

\section{RDW and proportion of intestinal resection in different activities subgroups}

The RDW in patients with different activities were 14.65 (2.48), 16.21 (3.41) and 17.61 (4.02), respectively. The $\mathrm{P}$ value between the moderate activity and the severe activity was 0.028 , but the $\mathrm{P}$ value was $<0.001$ between any other two groups (Figure 1). Of these, the proportion of patients undergoing intestinal resection was also statistically different in different activities groups. $20.7 \%$ were in the mild activity $(57 / 275), 36.5 \%$ were in the moderate activity $(23 / 63)$, and $46.4 \%$ were in the severe activity (13/28). And there were statistical differences between the three groups, $\mathrm{p}=0.001$, the chi-square value was 13.802 (Table 2).

\section{Correlations between RDW and baseline parameters}

RDW was significantly correlated with HGB $(\mathrm{r}=-0.629, \mathrm{p}<0.001)$, HCT $(\mathrm{r}=-0.566, \mathrm{p}<0.001)$, PLT $(\mathrm{r}=$ $0.385, \mathrm{p}<0.001), \operatorname{PDW}(\mathrm{r}=-0.253, \mathrm{p}<0.001), \mathrm{CRP}(\mathrm{r}=0.164, \mathrm{p}=0.008)$ (Figure 2$)$. However, there was no significant correlation between RDW and WBC $(\mathrm{r}=0.059, \mathrm{p}=0.26)$.

\section{Probability of surgery from diagnosis}

During the mean follow-up of 5 years, a total of 93 patients underwent intestinal resection, of which 89 patients with CD and 4 patients with UC. ROC curve analysis demonstrated that the optimal cutoff value of RDW for predicting intestinal resection was $13.95 \%$ with an area under the curve of $0.662(\mathrm{p}<0.001)$ (Figure 3 ). The sensitivity and specificity were $76.3 \%$ and $48.7 \%$, respectively. Kaplan-Meier analysis showed that patients with RDW [?]13.95\% had a significantly higher probability of intestinal resection than those with RDW $<13.95 \%(\mathrm{p}<0.001)$ (Figure 4). 


\section{Independent prognostic markers of surgery}

A univariate Cox regression analysis identified IBD type (CD or $\mathrm{UC})(\mathrm{P}<0.001)$, systolic blood pressure $(\mathrm{P}<0.001)$, RDW $(\mathrm{P}<0.001)$, plalet count $(\mathrm{P}=0.001)$, neutrophil ratio $(\mathrm{P}<0.001)$, lymphocyte ratio $(\mathrm{P}=0.002)$, C-reactive protein $(\mathrm{P}<0.001)$ and high-sensitivity $\mathrm{C}$-reactive protein $(\mathrm{P}=0.04)$ as significant prognostic factors of probability of intestinal resection. The multivariate analysis demonstrated that RDW (OR 1.088 (95\% CI 1.009-1.173), $\mathrm{P}=0.03)$ and IBD type $(\mathrm{P}=0.001)$ were independent predictors of intestinal resection probability in IBD (Table 3 ).

\section{Discussion}

Our study demonstrated that RDW reflects the severity of the disease and is associated with inflammatory indicators. Patients with severe disease often fail to receive conservative treatment and need surgical treatment. Our result further demonstrated that RDW and IBD type were independent predictors for intestinal resection.

Red cell distribution width (RDW) reflects the size variability of circulating red blood cells. It has shown promising potential as an index of disease activity and severity in various pathologic conditions ${ }^{13-17}$. A number of clinical studies have been performed to evaluate its application as a surrogate marker of disease activity in $\mathrm{IBD}^{5,7}, 8,18-20$. To the best of our knowledge, we firstly found RDW was significantly higher in intestinal resection compared with non-intestinal resection group. Furthermore, RDW was significantly correlated with CRP, hs-CRP and other inflammatory indicators, which was consistent with the study by Cakal B et al ${ }^{5}$. This study also showed that the patients with higher RDW were at a higher risk of receiving intestinal resection than those with lower RDW. Using multivariate Cox regression analysis, we finally found that RDW was an independent prognostic marker of intestinal resection probability in IBD. Our results are complementary to recent data that have demonstrated the value of RDW in evaluating the severity of disease in patients with IBD patients ${ }^{5}, 21$. Our results suggest the potential role of RDW in treatment selection. The exact mechanism of RDW associated with disease severity is unclear. There are several possible mechanisms. Firstly, The RDW may elevate in conditions of ineffective red cell production (iron deficiency, B12 or folate deficiency, and hemoglobinopathies), increased red cell destruction (hemolysis), or after blood transfusions ${ }^{22,23}$. Anemia caused by IBD may be one reason contribute to the elevation of RDW. In addition, IBD are chronic inflammatory conditions of the gastrointestinal tract ${ }^{24}$. Chronic inflammation may impair erythropoietin by the exertional effect of cytokines, such as interleukin-1, interleukin-6, tumor necrosis factor- $\alpha$, interferon- $\gamma$ - production and so on ${ }^{25,26}$. Finally, these events lead to the alterations of red blood cell life span, diminished responsiveness of bone marrow to erythropoietin and impaired iron metabolism, disrupting the normal process of hematopoiesis ${ }^{25,26}$. In summary, anemia and the direct or indirect effects of inflammation and oxidative stress may possibly explain the correlation of RDW with disease activity 27,28 .

Also, Nguyen GC suggested that diagnosable malnutrition was associated with greater mortality and worse outcomes $^{29}$. And Mańkowska-Wierzbicka D found a correlation between hs-CRP concentrations at diagnosis and risk of surgery in the group of CD patients, but not in the group of UC patients ${ }^{30}$. However, we found no such correlation in the present study, and a previous study support our results ${ }^{31}$.

Our study demonstrated that RDW was superior to CRP and other inflammatory indicators for the prediction of bowel resection probability. But it contains several limitations. First, information on the dynamic changes in RDW was lacking, and the data collected for each patient on admission may have been from different disease stages. Second, the sample size of our population was small. Thus, Large-scale well-designed studies are necessary to conduct to evaluate whether the limited but rather encouraging data are sufficient to establish RDW as a reliable activity index. Third, as a retrospective study, some other parameters which may predict prognosis were not done in all the patients.

We concluded that RDW is an independent predictor of bowel resection in IBD patients. Considering the cost-effective and popularity of RDW, It is worthy of clinical use. 


\section{Acknowledgments}

The study was funded by "The Fundamental Research Funds for the Central Universities (19ykpy10)".

\section{Contribution statement}

$\mathrm{XJ}, \mathrm{ZM}, \mathrm{ZY}$, and CS collected the epidemiological and clinical data. XJ and CZ processed statistical data. $\mathrm{XJ}, \mathrm{ZW}, \mathrm{ZM}$, and ZY drafted the manuscript. ZW, ZM and OY revised the final manuscript. All authors agree to be accountable for all aspects of the work in ensuring that questions related to the accuracy or integrity of any part of the work are appropriately investigated and resolved.

\section{References}

1. Abdelrazeq AS, Wilson TR, Leitch DL, et al. Ileitis in ulcerative colitis: is it a backwash? Diseases of the colon and rectum . Nov 2005;48(11):2038-46. doi:10.1007/s10350-005-0160-3

2. Rudolph WG, Uthoff SM, McAuliffe TL, et al. Indeterminate colitis: the real story. Diseases of the colon and rectum. Nov 2002;45(11):1528-34. doi:10.1007/s10350-004-6461-0

3. Gajendran M, Loganathan P, Catinella AP, Hashash JG. A comprehensive review and update on Crohn's disease. Disease-a-month : DM . Feb 2018;64(2):20-57. doi:10.1016/j.disamonth.2017.07.001

4. Biasci D, Lee JC, Noor NM, et al. A blood-based prognostic biomarker in IBD. Gut . Aug 2019;68(8):13861395. doi:10.1136/gutjnl-2019-318343

5. Cakal B, Akoz AG, Ustundag Y, et al. Red cell distribution width for assessment of activity of inflammatory bowel disease. Digestive diseases and sciences . Apr 2009;54(4):842-7. doi:10.1007/s10620-008-0436-2

6. Katsaros M, Paschos P, Giouleme O. Red cell distribution width as a marker of activity in inflammatory bowel disease: a narrative review.Annals of gastroenterology . Jul-Aug 2020;33(4):348-354. doi:10.20524/aog.2020.0486

7. Song CS, Park DI, Yoon MY, et al. Association between red cell distribution width and disease activity in patients with inflammatory bowel disease. Digestive diseases and sciences. Apr 2012;57(4):1033-8. doi:10.1007/s10620-011-1978-2

8. Yeşil A, Senateş E, Bayoğlu IV, et al. Red cell distribution width: a novel marker of activity in inflammatory bowel disease. Gut and liver . Dec 2011;5(4):460-7. doi:10.5009/gnl.2011.5.4.460

9. Li Y, Xing C, Wei M, et al. Combining Red Blood Cell Distribution Width (RDW-CV) and CEA Predict Poor Prognosis for Survival Outcomes in Colorectal Cancer. Journal of Cancer . 2019;10(5):1162-1170. doi:10.7150/jca.29018

10. Stange EF, Travis SP, Vermeire S, et al. European evidence based consensus on the diagnosis and management of Crohn's disease: definitions and diagnosis. Gut . Mar 2006;55 Suppl 1(Suppl 1):i1-15. doi:10.1136/gut.2005.081950a

11. D'Haens G, Sandborn WJ, Feagan BG, et al. A review of activity indices and efficacy end points for clinical trials of medical therapy in adults with ulcerative colitis. Gastroenterology . Feb 2007;132(2):763-86. doi:10.1053/j.gastro.2006.12.038

12. Schroeder KW, Tremaine WJ, Ilstrup DM. Coated oral 5-aminosalicylic acid therapy for mildly to moderately active ulcerative colitis. A randomized study. The New England journal of medicine. Dec 24 1987;317(26):1625-9. doi:10.1056/nejm198712243172603

13. Ephrem G, Kanei Y. Elevated red blood cell distribution width is associated with higher recourse to coronary artery bypass graft.Cardiology . 2012;123(3):135-41. doi:10.1159/000342268

14. He Y, Liu C, Zeng Z, et al. Red blood cell distribution width: a potential laboratory parameter for monitoring inflammation in rheumatoid arthritis. Clinical rheumatology. Jan 2018;37(1):161-167. 
doi:10.1007/s10067-017-3871-7

15. Kim CH, Park JT, Kim EJ, et al. An increase in red blood cell distribution width from baseline predicts mortality in patients with severe sepsis or septic shock. Critical care (London, England). Dec 9 2013;17(6):R282. doi:10.1186/cc13145

16. Kim HM, Kim BS, Cho YK, et al. Elevated red cell distribution width is associated with advanced fibrosis in NAFLD. Clinical and molecular hepatology . Sep 2013;19(3):258-65. doi:10.3350/cmh.2013.19.3.258

17. Wang H, Wang J, Huang R, et al. Red blood cell distribution width for predicting significant liver inflammation in patients with autoimmune hepatitis. European journal of gastroenterology 83 hepatology . Dec 2019;31(12):1527-1532. doi:10.1097/meg.0000000000001447

18. Ipek S, Cekic C, Alper E, et al. Can red cell distribution width be a marker of disease activity in ulcerative colitis? International journal of clinical and experimental medicine . 2015;8(8):13848-53.

19. Oliveira AM, Cardoso FS, Rodrigues CG, et al. Can Red Cell Distribution Width Be Used as a Marker of Crohn's Disease Activity?GE Portuguese journal of gastroenterology . Jan-Feb 2016;23(1):6-12. doi:10.1016/j.jpge.2015.10.003

20. Hu D, Ren J, Wang G, et al. Value of red cell distribution width for assessing disease activity in Crohn's disease. The American journal of the medical sciences . Jan 2015;349(1):42-5. doi:10.1097/maj.0000000000000334

21. Clarke K, Sagunarthy R, Kansal S. RDW as an additional marker in inflammatory bowel disease/undifferentiated colitis. Digestive diseases and sciences . Sep 2008;53(9):2521-3. doi:10.1007/s10620007-0176-8

22. Felker GM, Allen LA, Pocock SJ, et al. Red cell distribution width as a novel prognostic marker in heart failure: data from the CHARM Program and the Duke Databank. Journal of the American College of Cardiology . Jul 3 2007;50(1):40-7. doi:10.1016/j.jacc.2007.02.067

23. Tonelli M, Sacks F, Arnold M, et al. Relation Between Red Blood Cell Distribution Width and Cardiovascular Event Rate in People With Coronary Disease. Circulation . Jan 15 2008;117(2):163-168. doi:10.1161/circulationaha.107.727545

24. Zhang YZ, Li YY. Inflammatory bowel disease: pathogenesis. World journal of gastroenterology . Jan 7 2014;20(1):91-9. doi:10.3748/wjg.v20.i1.91

25. Weiss G, Goodnough LT. Anemia of chronic disease. The New England journal of medicine . Mar 10 2005;352(10):1011-23. doi:10.1056/NEJMra041809

26. Price EA, Schrier SL. Unexplained aspects of anemia of inflammation.Advances in hematology . 2010;2010:508739. doi:10.1155/2010/508739

27. Lippi G, Mattiuzzi C, Cervellin G. Learning more and spending less with neglected laboratory parameters: the paradigmatic case of red blood cell distribution width. Acta bio-medica : Atenei Parmensis . Jan 16 2016;87(3):323-328.

28. Zurauskaite G, Meier M, Voegeli A, et al. Biological pathways underlying the association of red cell distribution width and adverse clinical outcome: Results of a prospective cohort study. PloS one . 2018;13(1):e0191280. doi:10.1371/journal.pone.0191280

29. Nguyen GC, Munsell M, Harris ML. Nationwide prevalence and prognostic significance of clinically diagnosable protein-calorie malnutrition in hospitalized inflammatory bowel disease patients.Inflammatory bowel diseases . Aug 2008;14(8):1105-11. doi:10.1002/ibd.20429

30. Mańkowska-Wierzbicka D, Karczewski J, Poniedziałek B, et al. C-reactive protein as a diagnostic and prognostic factor in inflammatory bowel diseases. Postepy higieny i medycyny doswiadczalnej (Online). Nov 
7 2016;70(0):1124-1130. doi:10.5604/17322693.1223798

31. Bamba S, Sasaki M, Takaoka A, et al. Sarcopenia is a predictive factor for intestinal resection in admitted patients with Crohn's disease. PloS one . 2017;12(6):e0180036. doi:10.1371/journal.pone.0180036

Table 1 Baseline characteristics of patients with IBD and comparison between the intestinal resection group and the non- intestinal resection group

\begin{tabular}{|c|c|c|c|c|}
\hline Characteristic & Total $(\mathrm{n}=366)$ & $\begin{array}{l}\text { Intestinal resection } \\
\text { group }(\mathrm{n}=93)\end{array}$ & $\begin{array}{l}\text { Non-intestinal } \\
\text { resection group } \\
(\mathrm{n}=273)\end{array}$ & P-value \\
\hline Age, years & $30(22-41)$ & $30(23-37)$ & $30(22-42)$ & 0.21 \\
\hline Female, n(\%) & 33.1 & 39.8 & 30.8 & 0.11 \\
\hline $\mathrm{CD} / \mathrm{UC}, \mathrm{n}(\%)$ & $261 / 105$ & $89 / 4$ & $172 / 101$ & 0.001 \\
\hline $\mathrm{SBP}, \mathrm{mmHg}$ & $111(12)$ & $107(12)$ & $112(12)$ & 0.001 \\
\hline $\mathrm{DBP}, \mathrm{mmHg}$ & $68(9)$ & $66(9)$ & $68(9)$ & 0.07 \\
\hline $\mathrm{Cr}, \mu \mathrm{mol} / \mathrm{L}$ & $60.96(18.86)$ & $57.70(16.46)$ & $62.50(19.79)$ & 0.16 \\
\hline $\mathrm{UA}, \mu \mathrm{mol} / \mathrm{L}$ & $329.65(117.03)$ & $326.82(104.52)$ & $330.93(122.57)$ & 0.81 \\
\hline RDW, $\%$ & $15.15(2.94)$ & $16.20(3.24)$ & $14.76(2.70)$ & $<0.001$ \\
\hline PLT, 10E9/L & $315.64(118.50)$ & $347.67(131.42)$ & $304.73(111.94)$ & 0.005 \\
\hline $\mathrm{PDW}, \mathrm{fl}$ & $10.90(2.01)$ & $10.68(1.87)$ & $10.97(2.06)$ & 0.23 \\
\hline WBC, 10E9/L & $7.78(3.20)$ & $7.94(3.85)$ & $7.72(2.96)$ & 0.57 \\
\hline NEUR & $0.64(0.14)$ & $0.69(0.20)$ & $0.63(0.12)$ & 0.009 \\
\hline LYMR & $0.24(0.10)$ & $0.21(0.11)$ & $0.25(0.10)$ & 0.005 \\
\hline $\mathrm{CRP}, \mathrm{mg} / \mathrm{L}$ & $23.37(28.96)$ & $35.36(35.28)$ & $19.36(25.40)$ & 0.001 \\
\hline Hs-CRP, mg/L & $9.10(7.64)$ & $10.76(4.91)$ & $8.54(8.31)$ & 0.02 \\
\hline
\end{tabular}

Values are expressed as the mean (SD) or median (IQR).

Abbreviations: CD, Crohn's disease; UC, ulcerative colitis; SBP, systolic blood pressure; DBP, diastolic blood pressure; Cr, creatinine; UA, uric acid; RDW, red blood cell distribution width; PLT, platelet; PDW, platelet distribution width; WBC, white blood cells; NEUR, neutrophil ratio; LYMR, lymphocyte ratio; CRP, C-reactive protein; Hs-CRP, high-sensitivity C-reactive protein.

Table 2 Proportion of intestinal resection in different activities subgroups

\begin{tabular}{llll}
\hline Activities subgroups & Intestinal resection group & Non-intestinal resection & Total \\
\hline Mild activity & $57(20.7 \%)$ & $218(79.3 \%)$ & 275 \\
Moderate activity & $23(36.5 \%)$ & $40(63.5 \%)$ & 63 \\
Severe activity & $13(46.4 \%)$ & $15(53.6 \%)$ & 28 \\
\hline
\end{tabular}

Table 3 Univariate and multivariate Cox regression analyses of predictors of surgery in patients IBD

\begin{tabular}{lllll}
\hline Variable & Univariate Cox analysis & Univariate Cox analysis & Multivariate Cox analysis & Multivariate Cox analysi \\
\hline & $\mathrm{HR}(95 \% \mathrm{CI})$ & $\mathrm{p}$-value & HR $(95 \% \mathrm{CI})$ & $\mathrm{p}$-value \\
Age, years & $0.991(0.975-1.006)$ & 0.24 & - & - \\
Female, $\mathrm{n}(\%)$ & $0.726(0.749-1.101)$ & 0.13 & - & - \\
$\mathrm{CD} / \mathrm{UC}, \mathrm{n}(\%)$ & $10.703(3.929-29.154)$ & $<0.001$ & $0.130(0.041-0.416)$ & 0.001 \\
$\mathrm{SBP}, \mathrm{mmHg}$ & $0.967(0.950-0.985)$ & $<0.001$ & - & - \\
$\mathrm{DBP}, \mathrm{mmHg}$ & $0.977(0.953-1.002)$ & 0.07 & - & -
\end{tabular}




\begin{tabular}{lllll}
\hline Variable & Univariate Cox analysis & Univariate Cox analysis & Multivariate Cox analysis & Multivariate Cox analysi \\
\hline $\mathrm{Cr}, \mu \mathrm{mol} / \mathrm{L}$ & $0.989(0.972-1.006)$ & 0.22 & - & - \\
$\mathrm{UA}, \mu \mathrm{mol} / \mathrm{L}$ & $1.000(0.997-1.002)$ & 0.73 & - & - \\
$\mathrm{RDW}, \%$ & $1.116(1.059-1.176)$ & $<0.001$ & $1.088(1.009-1.173)$ & 0.03 \\
$\mathrm{PLT}, 10 \mathrm{E} 9 / \mathrm{L}$ & $1.003(1.001-1.004)$ & 0.001 & - & - \\
$\mathrm{PDW}, \mathrm{f}$ & $0.937(0.844-1.041)$ & 0.22 & - & - \\
$\mathrm{WBC}, 10 \mathrm{E} 9 / \mathrm{L}$ & $1.029(0.963-1.099)$ & 0.40 & - & - \\
$\mathrm{NEUR}$ & $5.462(2.337-12.767)$ & $<0.001$ & - & - \\
$\mathrm{LYMR}$ & $0.033(0.004-0.284)$ & 0.002 & - & - \\
$\mathrm{CRP}, \mathrm{mg} / \mathrm{L}$ & $1.010(1.005-1.016)$ & $<0.001$ & - & - \\
Hs-CRP, $\mathrm{mg} / \mathrm{L}$ & $1.016(1.001-1.032)$ & 0.04 & - & - \\
\hline
\end{tabular}

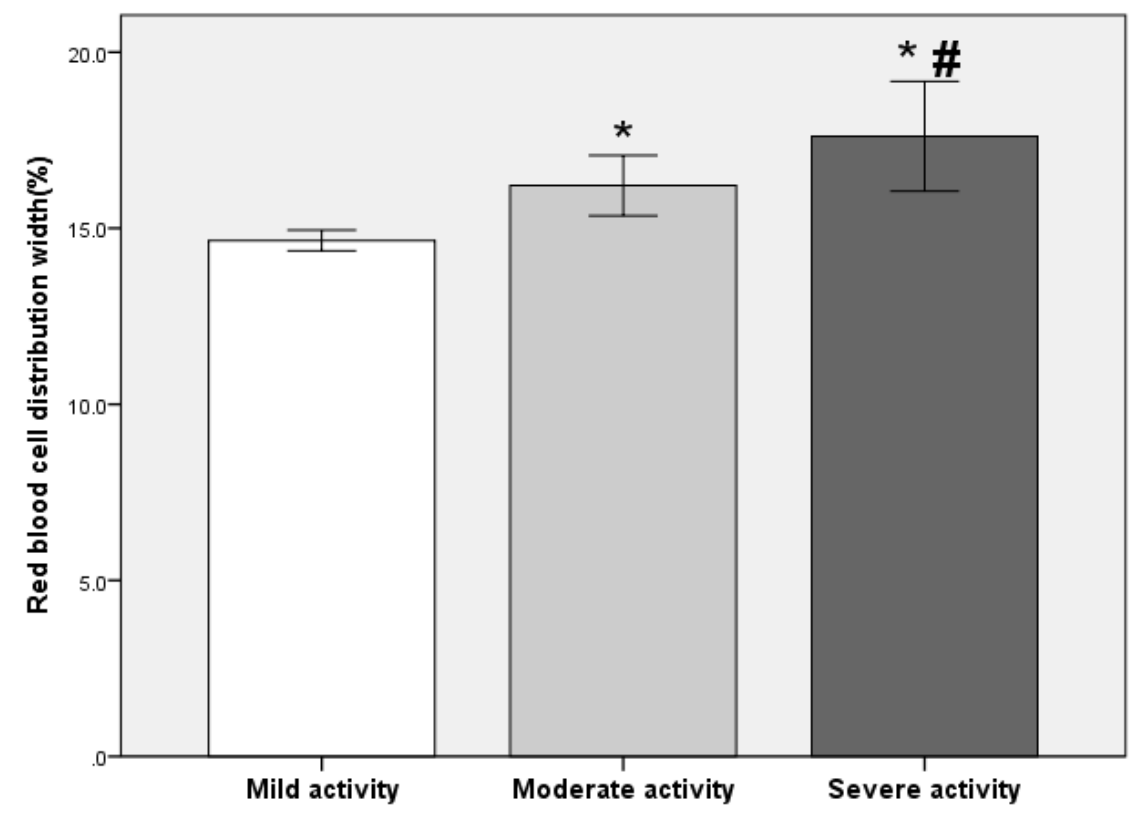

\section{Hosted file}

Figure 2.docx available at https://authorea.com/users/432493/articles/535982-prognosticvalue-of-red-blood-cell-distribution-width-for-intestinal-resection-in-inflammatorybowel-disease 

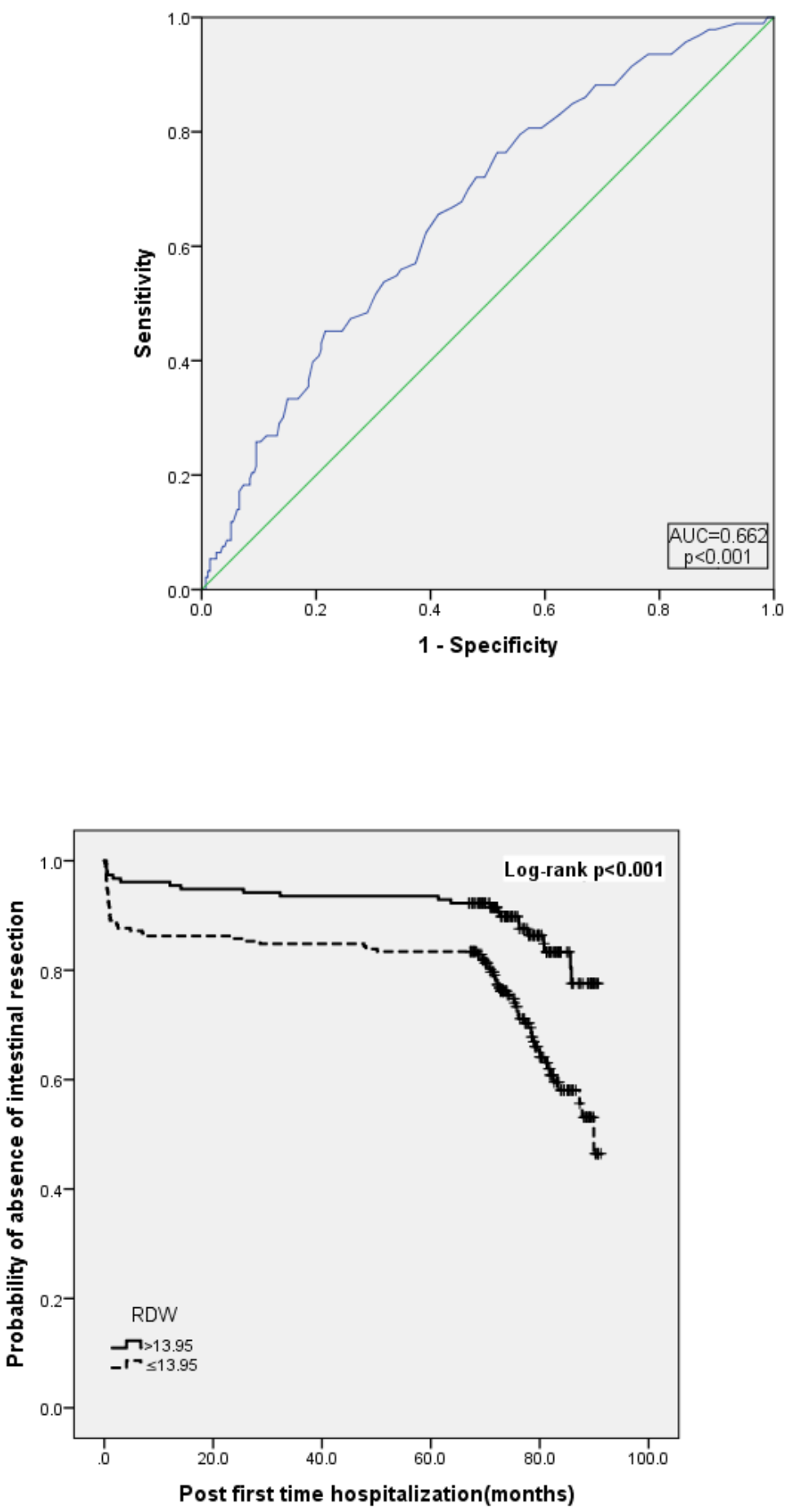